\title{
Haemolysis index - an estimate of preanalytical quality in primary health care
}

\author{
Johan Söderberg ${ }^{1, *}$, P. Andreas Jonsson ${ }^{1}$, Olof \\ Wallin $^{1,2}$, Kjell Grankvist ${ }^{1}$ and Johan Hultdin ${ }^{1}$ \\ ${ }^{1}$ Department of Medical Biosciences, Clinical \\ Chemistry, Umeå University, Umeå, Sweden \\ ${ }^{2}$ Strategic Activity Development, Karolinska \\ University Hospital, Stockholm, Sweden
}

\begin{abstract}
Background: Haemolysis is usually caused by inadequate specimen collection or preanalytical handling, and is suggested to be a suitable indicator of preanalytical quality. We investigated the prevalence of detectable haemolysis in all routine venous blood samples to identify differences in preanalytical quality.

Methods: Haemolysis index $(\mathrm{HI})$ values were obtained from a Vitros 5,1 in the routine clinical chemistry laboratory for samples collected in primary health care centres (PHCs), nursing homes, and a hospital emergency department (ED). Haemolysis was defined as a $\mathrm{HI} \geq 15$ (detection limit).

Results: Samples from the PHC with the highest prevalence of haemolysis were 6.1 times $(95 \%$ confidence interval (Cl) 4.0-9.2) more often haemolysed compared to the centre with the lowest prevalence. Of the samples collected in primary health care, $10.4 \%$ were haemolysed compared to $31.1 \%$ in the ED ( $p<$ 0.001). A notable difference in haemolysed samples was found between the ED section staffed by emergency medicine physicians and the section staffed by primary health care physicians $(34.8 \%$ vs. $11.3 \%$, $\mathrm{p}<0.001$ ).

Conclusions: The significant variation in haemolysis indices among the investigated units is likely to reflect varying preanalytical conditions. The $\mathrm{HI}$ is a valuable tool for estimation and follow-up of preanalytical quality in primary health care.

Clin Chem Lab Med 2009;47:940-4.
\end{abstract}

Keywords: blood specimen collection; haemolysis; medical errors; primary health care; quality indicators.

\section{Introduction}

Haemolysis is the release of haemoglobin and other intracellular components from erythrocytes into the

\footnotetext{
*Corresponding author: Johan Söderberg, Department of Medical Biosciences, Clinical Chemistry, Umeå University, Building 6M 2nd floor, 90185 Umeå, Sweden Phone: + 46-90-7852841, Fax: + 46-90-7854484, E-mail: johan.soderberg@medbio.umu.se Received April 7, 2009; accepted May 25, 2009 previously published online July 10, 2009
}

surrounding plasma following damage of the cell membrane (1). Haemolysis is a common reason for specimen rejection (2), reported to account for $40 \%-70 \%$ of all unsuitable specimens sent to the laboratory (1). The variation is dependent on different methods used for estimation of haemolysis, as well as different cut-off thresholds for analytical interference. A growing body of evidence indicates that most errors in laboratory testing arise in the preanalytical phase $(3,4)$ as the result of human mistakes (5). In vitro haemolysis is one important example since this is caused primarily by inappropriate specimen collection and handling (1), such as prolonged use of venous stasis (6), delayed separation of blood from plasma (1) and blood collection through intravenous catheters $(7,8)$.

We have previously reported unsatisfactory blood collection practices in primary health care centres (PHCs) $(9,10)$, where the majority of patient contact with caregivers occurs (11). This emphasises the importance of the preanalytical phase in the overall quality of the total testing process in PHCs. The prevalence of haemolysed specimens has been suggested as a suitable marker for preanalytical quality (12). Most previous studies have used subjective visual assessment $(6-8,13-15)$ or the analysis of free haemoglobin with laborious manual spectrophotometric techniques $(14,16-18)$ to evaluate the prevalence of haemolysis. The haemolysis index $(\mathrm{HI})$ in automated analysers is a more efficient method for detecting haemolysis. For many years now, the $\mathrm{HI}$ has been used in laboratories to automatically reject samples that are haemolysed in order to avoid analytical interference. However, the possible use of all samples with detectable $\mathrm{HI}$ as a marker of the overall preanalytical quality of the blood sample has not been reported previously.

The aim was to use $\mathrm{HI}$ as an automated determinant of haemolysis in venous blood samples sent from PHCs to the routine laboratory. We investigated the effect of different health care units, age and gender on the prevalence of haemolysis.

\section{Materials and methods}

\section{Subjects and setting}

The clinical chemistry laboratory in this study was located at a university hospital. The samples investigated were received from the neighbouring PHCs $(n=14)$ and nursing homes $(n=12)$, and the emergency department (ED) located in the university hospital.

The majority of samples from the PHCs were collected by enrolled nurses and, to a lesser extent, by registered nurses. The nursing homes were staffed with enrolled and registered 
nurses, and blood collection was performed by both staff categories. We compared two of the sections from the ED; the first staffed by emergency medicine physicians and the second staffed by PHC physicians. The latter handled patients with less critical conditions. The ED blood collection staff (registered and enrolled nurses) manned both sections on a rotating schedule. The distribution of blood collection staff in the investigated units is typical for Sweden. The laboratory manual was identical for all units investigated.

\section{Sample collection and measurements}

Routine venous blood samples were collected by use of a needle (and also by intravenous catheters in the ED) into plastic $3.5 \mathrm{~mL}$ evacuated serum separator test tubes with an inert polymer gel barrier and a clot activator (Becton Dickinson, Franklin Lakes, NJ, USA, cat-no 367957). After allowing the sample to clotting for $30 \mathrm{~min}$, the samples were centrifuged locally or in the laboratory, and then analysed for routine clinical chemistry analytes using a Vitros 5,1 automated analyser (Ortho-Clinical Diagnostics, Inc., Rochester, NY, USA). This analyser automatically measured $\mathrm{HI}$ using a spectrophotometric technique (19) in all blood samples. HI for the Vitros 5,1 was evaluated by serial dilution of a purified haemolysate into two serum samples with a low degree of haemolysis. The amount of free haemoglobin in these samples was measured using a spectrophotometric assay (20). There was a linear relationship between $\mathrm{HI}$ and the amount of free haemoglobin $\left(R^{2}=0.9865\right)$, and $1 \mathrm{~g} / \mathrm{L}$ of haemoglobin corresponded to a $\mathrm{HI}$ of 99 . The samples were considered haemolysed at an $\mathrm{HI} \geq 15$ (equivalent to $0.15 \mathrm{~g} / \mathrm{L}$ of free haemoglobin). This level was the lowest detectable value, and was chosen to compare the total amount of haemolysed samples from the units we investigated.

\section{Data collection}

We performed a retrospective study on samples $(n=9504)$ sent from PHCs, nursing homes and the university hospital
ED to the clinical chemistry laboratory for analysis on the Vitros 5,1 analyser (Table 1). Three identical analysers were used on a rotating schedule. $\mathrm{HI}$ values and the laboratory identification number (LID) were obtained from the analysers for samples analysed during the study period (May to September 2008). Information on age and gender of the patients along with the ordering unit was obtained from the laboratory information system (Flexlab 2.2.2, TietoEnator, Kista, Sweden).

The PHCs were divided according to their distance from the laboratory. Group one included eight PHCs with distances ranging from 1 to $8 \mathrm{~km}$ from the laboratory, and group two was comprised of the other six PHCs (distances ranging from 17 to $86 \mathrm{~km}$ ). Test tubes were collected in cooled insulated transport boxes $\left(5-12^{\circ} \mathrm{C}\right)$. They were collected twice a day from the PHCs close to the laboratory, and once a day from the more remote PHCs.

\section{Statistical analysis}

Data from the analysers and the laboratory information system were merged using the corresponding LID for each analysis. The complete file contained the analysis, the $\mathrm{HI}$ value, the ordering unit, and age and gender of the patient. Duplicate LIDs were excluded (i.e., samples where more than one analysis had been performed) resulting in a file containing one LID for each test tube. Cases with missing $\mathrm{HI}$ due to machine error $(n=191)$, or cases where invalid patient identification prohibited determination of patient age and gender $(n=5)$ were excluded. SPSS 14.0 for Windows (SPSS Inc., Chicago, IL, USA) was used for all statistical analyses. Categorical variables were compared using the $\chi^{2}$-test. Multivariate logistic regression analysis was used to study how different units, gender and age simultaneously affected the likelihood of samples having a $\mathrm{HI} \geq 15$. To measure association, odds ratios (OR) and their corresponding 95\% confidence intervals $(\mathrm{Cl})$ were used. The significance level was set at $p<0.05$. The research plan was approved by the Regional Ethical Review Board (D-No 07-13M).

Table 1 Absolute number $(n)$ and percentage $(\%)$ of test tubes with haemolysis index $(H I) \geq 15$ in the units we investigated. Included is the 95th percentile of the HI value.

\begin{tabular}{|c|c|c|c|}
\hline Primary health care centres & $\mathrm{n}$ & $\mathrm{HI} \geq 15, \%$ & HI 95th \\
\hline Total & 8849 & $10.4^{\mathrm{a}}$ & 19 \\
\hline Men & 3755 & $11.9^{b}$ & 20 \\
\hline Women & 5094 & 9.3 & 19 \\
\hline Below median age ( $<63$ years) & 4629 & $9.2^{\mathrm{c}}$ & 19 \\
\hline Above median age ( $\geq 63$ years) & 4220 & 11.8 & 20 \\
\hline \multicolumn{4}{|l|}{ Nursing homes } \\
\hline Total & 208 & 12.5 & 23 \\
\hline Men & 75 & 14.7 & 22 \\
\hline Women & 133 & 11.3 & 26 \\
\hline Below median age $(<84$ years) & 114 & 14.0 & 24 \\
\hline Above median age ( $\geq 84$ years) & 94 & 10.6 & 22 \\
\hline \multicolumn{4}{|l|}{ Emergency department } \\
\hline Total & 447 & $31.1^{\mathrm{a}}$ & 49 \\
\hline Men & 232 & 33.2 & 67 \\
\hline Women & 215 & 28.8 & 45 \\
\hline Below median age $(<54$ years $)$ & 230 & 30.0 & 62 \\
\hline Above median age ( $\geq 54$ years) & 217 & 32.3 & 48 \\
\hline \multicolumn{4}{|l|}{ All units } \\
\hline Total & 9504 & 11.4 & 21 \\
\hline Men & 4062 & $13.1^{b}$ & 21 \\
\hline Women & 5442 & 10.1 & 20 \\
\hline Below median age ( $<63$ years) & 4939 & $10.5^{\mathrm{d}}$ & 21 \\
\hline Above median age ( $\geq 63$ years) & 4565 & 12.4 & 21 \\
\hline
\end{tabular}

$\mathrm{p}$-Values estimated with $\chi^{2}$-test. Significant difference between; ${ }^{a}$ primary health care centres and the emergency department, $\mathrm{p}<0.001$; ${ }^{\mathrm{b}}$ men and women, $\mathrm{p}<0.001$; below or above the median age, $\mathrm{p}<0.001$; ${ }^{\mathrm{d}}$ below or above the median age, $\mathrm{p}<0.004$. 


\section{Results}

Baseline characteristics are presented in Table 1. Of the 8849 test tubes that were analysed from the PHCs, $10.4 \%$ had a $\mathrm{HI} \geq 15$ with a 95 th percentile of 19 . For samples sent from the PHCs, haemolysed samples (HI $\geq 15$ ) were more common among men $(11.9 \%)$ than among women $(9.3 \%)(p<0.001)$. Haemolysed samples also occurred more frequently in patients above the median age than below the median age $(11.8 \%$ vs. $9.2 \%)(p<0.001)$. Univariate analysis showed that for the PHCs investigated, samples collected from men were haemolysed 1.3 times $(95 \% \mathrm{Cl} 1.1-1.5)$ as often compared to samples collected from women. Further, samples collected from patients above the median age (63 years) were haemolysed 1.2 times (95\% Cl 1.1-1.4) more often compared to samples from patients below the median age. There was no significant difference in the prevalence of haemolysed samples with respect to age or gender in nursing homes or in the ED. The PHCs had fewer haemolysed samples compared to samples sent from the ED $(10.4 \%$ vs. $31.1 \%, p<0.001)$. There was no significant difference in haemolysed samples between the PHCs and the nursing homes.

Multivariate associations are presented in Table 2. Major differences in the prevalence of haemolysed samples existed between the different PHCs. Samples collected in the PHC with the highest prevalence of haemolysed samples were 6.1 times (95\% Cl 4.0-9.2) more often haemolysed compared to the centre with the lowest prevalence. Further, PHCs located outside the urban area (more than $17 \mathrm{~km}$ from the laboratory) were haemolysed 1.7 times $(95 \% \mathrm{Cl} 1.5-2.0)$ more often compared to the PHCs close to the laboratory. A notable difference in haemolysed samples were found between the ED section staffed by emergency medicine physicians $(34.8 \%)$, and the section staffed by PHC physicians $\left(11.3 \%, \chi^{2}\right.$-test: $\left.p<0.001\right)$. Samples collected in the emergency medicine physician section of the ED were haemolysed 4.3 times $195 \% \mathrm{Cl}$ 2.0-9.4) more often compared to samples collected in the PHC physician section. There was no significant difference in the prevalence of haemolysed samples between the ED section staffed with PHC physicians and the PHCs (data not shown).

Rejection levels and percentage of possible rejected samples are presented in Table 3 . Of the samples from the PHCs that were investigated, 3.1\% had a $\mathrm{HI}$ value above 25 and $0.8 \%$ had an $\mathrm{HI}$ value above 50 . In the ED, the $\mathrm{HI}$ value exceeded 25 in one out of six samples $(16.1 \%)$, and exceeded 50 in $5.1 \%$ of all samples. These are levels at which the results of several routine assays would be rejected.

\section{Discussion}

The results of this study indicate that there is a significant variation in the prevalence of haemolysed samples among PHCs. Most cases of haemolysis in blood specimens are caused by mistakes in the collection and handling of the sample (1). The demonstrated differences between different PHCs were adjusted for age and gender. Thus, the differences are likely to be a reflection of varying preanalytical conditions, rather than variation in these patient characteristics. The higher prevalence of haemolysis in samples collected outside the urban area likely reflects differences in the collection and handling of the sample before centrifugation.

Samples from patients over the median age were associated with a higher prevalence of haemolysis in the PHCs. This finding is in contrast to a previous hospital report (21) where no age related differences in haemolysis were found. Difficulties in accessing veins, or repeated attempts at venipuncture, can cause haemolysis (15). Such difficulties can also lead to collection of blood from sites other than the preferred antecubital veins, resulting in haemolysis 16 , $13,21)$. Therefore, one possible explanation for the association between age and $\mathrm{HI}$ is due to greater difficulties in obtaining venous access in the elderly. We have no explanation for the differences the incidence of haemolysed specimens between men and women.

There was no difference in haemolysed samples between PHCs and nursing homes, suggesting similar preanalytical conditions. In line with previous findings, the ED stood out as a major contributor of haemolysed samples $(6,22)$. In addition, the ED section staffed with emergency medicine physicians had a higher prevalence of haemolysed samples when compared with the ED section staffed with PHC physicians. These results were independent of age and gender. The most probable explanation is a more common use of intravenous catheters for collection of blood samples in the emergency medicine section of the ED, a practice known to cause haemolysis (7, $8,15)$. Since the blood collection staff manned both sections, the differences seem less likely to depend on the skills and experience of the staff, which previously has been shown to affect specimen quality (23).

In vitro haemolysis is a major cause of rejection of specimens in laboratories (2). Specimen rejection is costly and repeat sampling is stressful for patients (24). Haemolysis can cause prolonged turn around time for the tests that are ordered. This could affect patient workflow and be harmful to critically ill patients (25). Further, undetected haemolysis can increase or decrease the values for several analytes $(17,18,26)$ leading to clinically significant alterations of, for example, potassium and lactate dehydrogenase (18). In fact, mild haemolysis, almost undetectable by visual inspection, is known to influence test results (18). In our study, more than $10 \%$ of all specimens, and $31 \%$ of the specimens from the ED, suffered from some degree of haemolysis. This is a higher percentage than previously reported for all samples $(2.6 \%-3.3 \%)$ and ED samples $(12.4 \%-18.1 \%)(6,8,16$, 22 . This difference could be due to the low detection limit for haemolysis used in this study. Subjective visual inspection, used in many of the previous reports, can be unreliable in the assessment of sam- 
Table 2 Absolute number $(\mathrm{n})$ and percentage (\%) of test tubes with haemolysis index $(\mathrm{HI}) \geq 15$ in the units investigated. The data are shown as multivariate models with odds ratios $(\mathrm{OR})$ and confidence intervals $(\mathrm{Cl})$.

\begin{tabular}{|c|c|c|c|c|}
\hline Unit $^{\mathrm{a}}$ & $\mathrm{n}$ & $\mathrm{HI} \geq 15 \%$ & $\mathrm{HI} \geq 15 \mathrm{OR}(95 \% \mathrm{Cl})$ & $\mathrm{p}$-Value \\
\hline Primary health care centres & 8849 & 10.4 & 1.0 & \\
\hline Nursing homes & 208 & 12.5 & $1.13(0.74-1.73)$ & 0.560 \\
\hline Emergency department & 447 & 31.1 & $3.93(3.17-4.86)$ & $<0.001$ \\
\hline \multicolumn{5}{|l|}{ Primary health care centres ${ }^{a}$} \\
\hline 1 & 574 & 6.6 & 1.0 & \\
\hline 2 & 963 & 6.6 & $1.00(0.66-1.52)$ & 0.999 \\
\hline 3 & 492 & 6.7 & $1.01(0.63-1.64)$ & 0.956 \\
\hline 4 & 443 & 6.8 & $0.97(0.59-1.60)$ & 0.916 \\
\hline 5 & 598 & 8.4 & $1.22(0.79-1.90)$ & 0.368 \\
\hline 6 & 1096 & 8.5 & $1.28(0.87-1.90)$ & 0.211 \\
\hline 7 & 731 & 8.6 & $1.39(0.91-2.11)$ & 0.128 \\
\hline 8 & 1425 & 9.1 & $1.40(0.96-2.04)$ & 0.079 \\
\hline 9 & 622 & 12.4 & $2.04(1.36-3.07)$ & 0.001 \\
\hline 10 & 484 & 14.9 & $2.44(1.61-3.69)$ & $<0.001$ \\
\hline 11 & 790 & 15.8 & $2.53(1.73-3.71)$ & $<0.001$ \\
\hline 12 & 25 & 16.0 & $2.71(0.88-8.30)$ & 0.082 \\
\hline 13 & 333 & 16.8 & $2.80(1.81-4.34)$ & $<0.001$ \\
\hline 14 & 273 & 31.9 & $6.06(3.99-9.21)$ & $<0.001$ \\
\hline \multicolumn{5}{|l|}{ Nursing homes ${ }^{a}$} \\
\hline 1 & 37 & 2.7 & 1.0 & \\
\hline 2 & 16 & 6.3 & $2.42(0.14-41.76)$ & 0.544 \\
\hline 3 & 14 & 7.1 & $3.65(0.21-64.90)$ & 0.378 \\
\hline 4 & 23 & 8.7 & $3.66(0.30-44.02)$ & 0.307 \\
\hline 5 & 28 & 10.7 & $5.84(0.55-62.50)$ & 0.144 \\
\hline 6 & 16 & 11.8 & $5.53(0.46-67.20)$ & 0.179 \\
\hline 7 & 20 & 15.0 & $6.49(0.59-71.70)$ & 0.127 \\
\hline 8 & 5 & 20.0 & $10.51(0.53-209.7)$ & 0.124 \\
\hline 9 & 5 & 20.0 & $8.20(0.41-164.1)$ & 0.169 \\
\hline 10 & 8 & 25.0 & $17.05(1.25-232.6)$ & 0.033 \\
\hline 11 & 35 & 25.7 & $16.30(1.86-142.9)$ & 0.012 \\
\hline 12 & 1 & 100.0 & $0.00(0.00-0.00)$ & 1.000 \\
\hline \multicolumn{5}{|l|}{ Emergency department sections ${ }^{a}$} \\
\hline $\begin{array}{l}\text { Staffed with primary health care } \\
\text { physicians }\end{array}$ & 71 & 11.3 & 1.0 & \\
\hline $\begin{array}{l}\text { Staffed with emergency medicine } \\
\text { physicians }\end{array}$ & 376 & 34.8 & $4.32(1.99-9.38)$ & $<0.001$ \\
\hline \multicolumn{5}{|l|}{ Distance to the laboratory ${ }^{a}$} \\
\hline $\begin{array}{l}8 \text { PHCs close to the } \\
\text { laboratory }(1-8 \mathrm{~km})\end{array}$ & 5903 & 8.4 & 1.0 & \\
\hline $\begin{array}{l}6 \text { PHCs far from the } \\
\text { laboratory }(17-86 \mathrm{~km})\end{array}$ & 2921 & 14.4 & $1.74(1.51-2.00)$ & $<0.001$ \\
\hline
\end{tabular}

${ }^{\mathrm{a} O R s}$ adjusted for age and gender. PHC, primary health care centre.

Table 3 Percentage (\%) of potentially rejected samples at the units investigated based on haemolysis index (HI) levels for specimen rejection according to Ortho-Clinical Diagnostics and the definition of mild haemolysis ( $>0.6 \mathrm{~g} / \mathrm{L})$ according to Lippi et al. (1).

\begin{tabular}{lccc}
\hline Unit & \multicolumn{2}{l}{ Percentage $(\%)$ of rejected samples } \\
\cline { 2 - 4 } & $\mathrm{HI} \geq 25^{\mathrm{a}}(0.25 \mathrm{~g} / \mathrm{L})^{\mathrm{c}}$ & $\mathrm{HI} \geq 50^{\mathrm{b}}(0.5 \mathrm{~g} / \mathrm{L})^{\mathrm{c}}$ & $\mathrm{HI} \geq 60(0.6 \mathrm{~g} / \mathrm{L})^{\mathrm{c}}$ \\
\hline Primary health care centres & 3.1 & 0.8 & 0.6 \\
Nursing homes & 4.8 & 1.4 & 0.4 \\
Emergency department & 16.1 & 5.1 & 4.6 \\
All units & 3.7 & 1.0 & 0.8 \\
\hline
\end{tabular}

Rejection levels for: ${ }^{a}$ Haptoglobin; ${ }^{b}$ routine assays including potassium, aspartate aminotransferase, alanine aminotransferase, alkaline phosphatase, iron, magnesium, lactate dehydrogenase, phosphate; 'corresponding levels of free haemoglobin.

ple haemolysis (27) when compared to the $\mathrm{HI}$, used in this study (28).

To our knowledge, this is the first study to survey $\mathrm{HI}$ in blood samples sent from PHCs, and to compare the $\mathrm{HI}$ with respect to type of unit. This study is also the first to assess the use of $\mathrm{HI}$ as a marker of the overall preanalytical quality of the blood sample. Using the lowest detectable $\mathrm{HI}$ level, we could study the total number of samples with haemolysis from the units we investigated, making it useful as a sensitive marker for preanalytical quality. This $\mathrm{HI}$ level $(\geq 15)$ is not intended to be used for sample rejection due to 
haemolysis. It must be noted that this level is lower than the cut-offs for rejection of each analyte based on $\mathrm{HI}$ levels set by the manufacturer.

The number of samples we investigated was high. The blood collection staff was representative for the respective setting in Sweden, and comparable to other blood collection staff categories internationally. Future studies are needed to investigate the influence of specific preanalytical practices on HI. A practical value of this use of the $\mathrm{HI}$ is to implement the correct quality interventions of all aspects of the preanalytical phase. It also allows the possibility for follow-up of such interventions.

In conclusion, the significant variation in $\mathrm{HI}$ among the investigated units is likely to reflect varying preanalytical conditions. Therefore, monitoring of haemolysis indices is a valuable tool for estimating preanalytical quality in primary health care.

\section{Conflict of interest disclosures}

None declared.

\section{Acknowledgements}

We would like to thank the Faculty of Medicine, Umeå University, Umeå, Sweden and Västerbotten County Council in Sweden for research funding.

\section{References}

1. Lippi G, Blanckaert N, Bonini P, Green S, Kitchen S, Palicka $\mathrm{V}$, et al. Haemolysis: an overview of the leading cause of unsuitable specimens in clinical laboratories. Clin Chem Lab Med 2008;46:764-72.

2. Jones BA, Calam RR, Howanitz PJ. Chemistry specimen acceptability: a College of American Pathologists QProbes study of 453 laboratories. Arch Pathol Lab Med 1997;121:19-26.

3. Kalra J. Medical errors: impact on clinical laboratories and other critical areas. Clin Biochem 2004;37:1052-62.

4. Carraro P, Plebani M. Errors in a stat laboratory: types and frequencies 10 years later. Clin Chem 2007;53:133842.

5. Wiwanitkit $V$. Types and frequency of preanalytical mistakes in the first Thai ISO 9002:1994 certified clinical laboratory, a 6-month monitoring. BMC Clin Pathol 2001; $1: 5$.

6. Burns ER, Yoshikawa N. Hemolysis in serum samples drawn by emergency department personnel versus laboratory phlebotomists. Lab Med 2002;33:378-80.

7. Grant MS. The effect of blood drawing techniques and equipment on the hemolysis of ED laboratory blood samples. J Emerg Nurs 2003;29:116-21.

8. Kennedy C, Angermuller S, King R, Noviello S, Walker J, Warden $\mathrm{J}$, et al. A comparison of hemolysis rates using intravenous catheters versus venipuncture tubes for obtaining blood samples. J Emerg Nurs 1996;22: 566-9.
9. Söderberg J, Brulin C, Grankvist K, Wallin O. Preanalytical errors in primary healthcare: a questionnaire study of information search procedures, test request management and test tube labelling. Clin Chem Lab Med 2009; 47:195-201.

10. Söderberg J, Wallin O, Grankvist K, Brulin C. Is the test result correct? A questionnaire study of blood collection practices in primary health care. J Eval Clin Pract. In press 2009.

11. Green LA, Fryer GE Jr, Yawn BP, Lanier D, Dovey SM. The ecology of medical care revisited. N Engl J Med 2001;344:2021-5.

12. Kirchner MJ, Funes VA, Adzet CB, Clar MV, Escuer MI, Girona JM, et al. Quality indicators and specifications for key processes in clinical laboratories: a preliminary experience. Clin Chem Lab Med 2007;45:672-7.

13. Fang L, Fang SH, Chung YH, Chien ST. Collecting factors related to the haemolysis of blood specimens. J Clin Nurs 2008;17:2343-51.

14. Cox SR, Dages JH, Jarjoura D, Hazelett S. Blood samples drawn from IV catheters have less hemolysis when 5-mL (vs. 10-mL) collection tubes are used. J Emerg Nurs 2004;30:529-33.

15. Dugan L, Leech L, Speroni KG, Corriher J. Factors affecting hemolysis rates in blood samples drawn from newly placed IV sites in the emergency department. J Emerg Nurs 2005;31:338-45.

16. Carraro P, Servidio G, Plebani M. Hemolyzed specimens: a reason for rejection or a clinical challenge? Clin Chem 2000;46:306-7.

17. Lippi G, Montagnana M, Salvagno GL, Guidi GC. Interference of blood cell lysis on routine coagulation testing. Arch Pathol Lab Med 2006;130:181-4.

18. Lippi G, Salvagno GL, Montagnana M, Brocco G, Guidi GC. Influence of hemolysis on routine clinical chemistry testing. Clin Chem Lab Med 2006;44:311-6.

19. Knabbe C, Ratge D, Christen K, Sonntag O. Evaluation of sample integrity measurement with VITROS 5, 1 FS chemistry system. J Lab Med 2009;33:33-8.

20. Kahn SE, Watkins BF, Bermes EW Jr. An evaluation of a spectrophotometric scanning technique for measurement of plasma hemoglobin. Ann Clin Lab Sci 1981;11: 126-31.

21. Tanabe $P$, Kyriacou DN, Garland F. Factors affecting the risk of blood bank specimen hemolysis. Acad Emerg Med 2003;10:897-900.

22. Pretlow L, Gandy T, Leibach EK, Russell B, Kraj B. A quality improvement cycle: hemolyzed specimens in the emergency department. Clin Lab Sci 2008;21:219-24.

23. Iversen LH. Pre-analytical variation in the measurements of sensitive markers of coagulation and fibrinolysis: the influence of venipuncture and mixing of blood. Haemostasis 1997;27:119-24.

24. Chyun D. Patients' perceptions of stressors in intensive care and coronary care units. Focus Crit Care 1989;16: 206-11.

25. Bonini P, Plebani M, Ceriotti F, Rubboli F. Errors in laboratory medicine. Clin Chem 2002;48:691-8.

26. Sonntag O. Haemolysis as an interference factor in clinical chemistry. J Clin Chem Clin Biochem 1986;24: 127-39.

27. Glick MR, Ryder KW, Glick SJ, Woods JR. Unreliable visual estimation of the incidence and amount of turbidity, hemolysis, and icterus in serum from hospitalized patients. Clin Chem 1989;35:837-9.

28. Hawkins R. Discrepancy between visual and spectrophotometric assessment of sample haemolysis. Ann Clin Biochem 2002;39:521-2. 